\title{
Age-period-cohort analysis of cervical cancer incidence in Taiwan: differences between squamous cell carcinoma and adenocarcinoma
}

P. D. WANG* \& R.S. LIN†

${ }^{*}$ Taipei Wanhwa District Health Center, Taipei; $\uparrow$ College of Public Health, National Taiwan University, Taipei, Taiwan

\begin{abstract}
Wang PD, Lin RS. Age-period-cohort analysis of cervical cancer incidence in Taiwan: differences between squamous cell carcinoma and adenocarcinoma. Int J Gynecol Cancer 1997; 7: 106-111.
\end{abstract}

To examine the etiologic distinction between squamous cell carcinoma and adenocarcinoma of the cervix, the relationship between the incidence of both histotypes of cancer and age at diagnosis, time period at diagnosis and birth cohort was analyzed using data from the Taiwan Cancer Registry. Included in the study were all cases of both histotypes occurring during the period 1979-90 within a population of Taiwanese women aged 25 to 75 years. A log-linear model modified from the method of Osmond and Gardner was used for the analyses. Age-periodcohort analyses of age effect indicated that, prior to the age group of 4951 , there is an almost identical incidence of both histotypes with an approximate linear trend of age effect but that, after this time, there is a divergence, with the age effect of increase slightly declining with age for squamous cell carcinoma and clearly declining for adenocarcinoma. In regard to period and cohort effects, a substantial moderation of squamous cell carcinoma risk was exhibited with both advancing recent calendar periods and birth cohorts, while adenocarcinoma risk also has a moderation but to a lesser extent. The model also identified the changes in female sex hormones after the menopause as a determinant of the differing age effects, the efficiency of Pap smear screening practices as a determinant of the differing period effects, and changes in reproductive patterns as a determinant of the differing cohort effects. These findings may provide clues with which to develop etiologic hypotheses and support the contention of etiologic distinctions between both histologic types.

KEYWORDS: adenocarcinoma of the cervix, age-period-cohort analysis, squamous cell carcinoma of the cervix.

Squamous cell carcinoma and adenocarcinoma are the two most common cervical malignancies. Although recent morphologic studies have demonstrated that squamous cell carcinoma of the cervix frequently coexists with cervical adenocarcinoma ${ }^{(1,2)}$, nevertheless there is substantial evidence to suggest that etiologic distinctions exist between the histologic types ${ }^{(3,4)}$.

Address for correspondence: Dr PD Wang, Wanhwa District Health Center, No. 152, Tung-Yuan Street, Taipei, Taiwan.
It has been proposed that there is no one lifestyle or epidemiologic characteristic that predisposes a patient to develop one histologic type of cervical cancer or another ${ }^{(5)}$.

Epidemiologic evidence for etiologic distinctions includes differences in time trends and in ageincidence curves for both histologic types. In reference to time trends, several studies from diverse geographical areas, including $\mathrm{USA}^{(6,7)}, \mathrm{UK}^{(8)}$ and Norway ${ }^{(4)}$, have demonstrated a significant decrease in the 
incidence of squamous cell carcinoma over the past several decades, whereas a trend to an increasing incidence of adenocarcinoma was observed during the same time period. In addition, in examining age trends using incidence data, Eide ${ }^{(4)}$ found that the rate of increase with age is almost identical for both histologic types prior to age 34 but that, subsequently, squamous cell carcinoma exhibits a moderate decline in the oldest part of the population whereas the incidence rates for adenocarcinoma were about the same for all ages above 45 years. Such trends, however, were not observed by Anton-Culver et al. ${ }^{(9)}$, who found continued rates of increase with age for both histologic types.

However previous analyses of time trends and ageincidence curves among the histologic types of the uterine cervix have not considered the simultaneous effects of age at diagnosis, time period at diagnosis, and birth cohort. To differentiate the effects due to age, period, and cohort, a log-linear Poisson model (the age-period-cohort (APC) model) has been successfully applied to analyze the secular trends of various diseases $^{(10-12)}$. Such APC analysis of cancer incidence data can provide useful clues related to cancer etiology ${ }^{(13)}$. Nevertheless, it is well-known that such a model suffers from an identifiability problem due to the exact linear dependence between age, period and cohort (cohort $=$ period-age). Although several methods have been proposed to solve the problem of nonidentifiability inherent in the APC model by introducing suitable constraints on the parameters in the model $^{(14,15)}$, the method of Osmond and Gardner ${ }^{(16)}$ was adopted in the present study since it does allow for quantifing the separate effect of the three factors: age, period and cohort.

This study applied a log-linear Poisson regression approach to analyze the relationship between squamous cell carcinoma and adenocarcinoma incidences and age, time period and birth cohort, using data from the Taiwan Cancer Registry. The differences in age-periodcohort patterns for both histological types are discussed.

\section{Patients and methods}

\section{Incidence data and population}

The National Cancer Registry Campaign was started only recently in Taiwan and hence information about the incidence of squamous cell carcinoma and adenocarcinoma of the cervix was available for this study only from 1979. Data of all squamous cell carcinoma and adenocarcinoma cases from 1979 through 1990 were obtained from the Taiwan Cancer Registry ${ }^{(17)}$ : each case was characterized by detailed demographic data including age at diagnosis, period of diagnosis and birth date. Cervical cancers reported to Taiwan Cancer Registry were classified into three histologic types according to the International Classification of Disease for Oncology (ICD-O) ${ }^{(18)}$ : squamous cell carcinoma (ICD-O codes 8000, 8010, 8070-8072 and 8076); adenocarcinoma (ICD-O codes 8140, 8260, 8430, 8441, 8480, 8481 and 8560); adenosquamous cell carcinoma (ICD-O codes 8560 and 8570). Data from a total of 18888 women with squamous cell carcinoma and 1500 women with adenocarcinoma aged 25-75 years were collected for the analysis during the period 1979-90. The incidence rates were classified by 3-year age groups. Age specific mid-year population estimates were obtained from data published by the Ministry of the Interior in Taiwan ${ }^{(19)}$.

\section{Statistical age-period-cohort analysis}

The matrix of the age-specific incidence of both histologic types was calculated for each 3-year period interval beginning with 1979-81, and 3-year age intervals, beginning with age 25-27. The effect of age, period and birth cohort were examined using a log-linear Poisson regression model, modified from Osmond and Gardner's method ${ }^{(16)}$. The statistical model used in these analyses was

$$
\log \left[R_{\mathrm{ijk}}\right]=K+A_{\mathrm{i}}+P_{\mathrm{j}}+C_{\mathrm{k}}+E_{\mathrm{ijk}}
$$

where $R_{\mathrm{ijk}}$ represents the observed incidence rate in a particular age-period-cohort category, $K$ is a constant, $A_{\mathrm{i}}, P_{\mathrm{j}}$ and $C_{\mathrm{k}}$ represent the age, period and cohort effects, respectively and $E_{\mathrm{ijk}}$ represents random error. The incidence of both histologic types is assumed to follow a Poisson distribution.

The estimates derived from the model, including the three time factors, that minimized the weighted sum of the Euclidean distances from the three possible twofactor model (age/period; age/cohort; period/cohort) based on the goodness-of-fit of each one. In the study, these measures were taken as the inverse of the deviance statistics. The sum of each of the three effects was constrained to be zero. These effects can be interpreted as logarithms of relative risks. The computer program SAS/IML was used for the computation ${ }^{(20)}$.

\section{Results}

During the period 1979-90, data from 18888 women aged 25-75 years with squamous cell carcinoma and 1500 women with adenocarcinoma were used for the analysis. Table 1 shows the rates of cervical cancer by 
Table 1. Incidence of cervical cancer (number of cases in parentheses) by histological type, age, and time period, Taiwan areas, 1979-90*

\begin{tabular}{|c|c|c|c|c|c|}
\hline \multirow[b]{2}{*}{ Histologic type } & \multicolumn{4}{|l|}{ Age } & \multirow[b]{2}{*}{ All ages } \\
\hline & $25-34$ & $35-49$ & $50-64$ & $65+$ & \\
\hline \multicolumn{6}{|c|}{ Squamous cell carcinoma } \\
\hline 1979-82 & $1.23(301)$ & 42.61 (2134) & $83.86(2628)$ & $56.76(869)$ & $17.41(5932)$ \\
\hline 1983-86 & 1.56 (399) & $42.57(2347)$ & $90.73(3283)$ & 70.52 (1269) & $20.03(7298)$ \\
\hline \multirow[t]{2}{*}{ 1987-90 } & $1.36(346)$ & $25.01(1600)$ & $61.63(2531)$ & 51.95 (1121) & $14.74(5658)$ \\
\hline & $5.7^{* *}\left(0.02^{* * *}\right)$ & $87.80\left(0.00^{* * *}\right)$ & $98.70\left(0.00^{* * *}\right)$ & $4.60\left(0.03^{* * *}\right)$ & $39.50\left(0.00^{* * *}\right)$ \\
\hline \multicolumn{6}{|l|}{ Adenocarcinoma } \\
\hline 1979-82 & $0.14(34)$ & $3.23(162)$ & $4.98(156)$ & $3.40(52)$ & $1.19(404)$ \\
\hline 1983-86 & $0.22(56)$ & 3.45 (190) & $5.97(216)$ & $5.22(94)$ & $1.53(556)$ \\
\hline \multirow[t]{2}{*}{ 1987-90 } & $0.23(57)$ & 2.79 (185) & $5.65(232)$ & $3.06(66)$ & $1.41(540)$ \\
\hline & $4.76\left(0.05^{* * *}\right)$ & $2.20(0.14)$ & $1.20(0.28)$ & $0.7(0.40)$ & $5.4\left(0.02^{* * *}\right)$ \\
\hline
\end{tabular}

*Rate per 100,000 women per year; ${ }^{* *} X^{2}$ value for trend-test ( $P$-value); ${ }^{* * *}$ significant at $\alpha=0.05$ level.

age, time period, and histologic type. It can be seen from the table that the incidence rates of squamous cell carcinoma appears to decrease in all age groups, while adenocarcinoma seems to increase from 1.19 to 1.41 per 100000 between 1979 and 1990, the increase being more pronounced among the age group of 25-34. However this analysis of age-specific incidence rates does not consider the period and cohort effect upon which the secular trend depended.

Figure 1 shows for squamous neoplasms and adenocarcinomas the logarithm of relative risk of cervical cancer incidence in different age groups (ie, the age effects) as derived from the APC model. For squamous cell carcinoma and adenocarcinoma, each appear almost identical with a nearly linear trend of age effect on both histologic types incidence prior to the age group of 49-51. Subsequently, however, there is a divergence, with the age effect of increase slightly declining with age for squamous cell carcinoma and clearly declining with increasing age for adenocarcinoma. The peak of the age effect for both histologic types occurs at the same age group of 49-51. The 49-51 age group has 1259 and 413 times the risk of the 25-27 age group for squamous cell carcinoma and adenocarcinoma, respectively. Thus the age effect on both histologic types appears very striking.

Figure 2 shows the period effect, presented also in the form of the logarithm of relative risks. There was a substantial decrease in the risk of squamous cell carcinoma as the period moved from 1979-81 to 1988-90, while the period effects for adenocarcinoma also decreased but to lesser extent during the period from 1985-87 through 1988-90. It is of interest to note that period effect was less striking than age effect for both histologic types.

As regards cohort effects, Fig. 3 displays the cohort effect from 1909-60. For the earlier birth cohorts, the

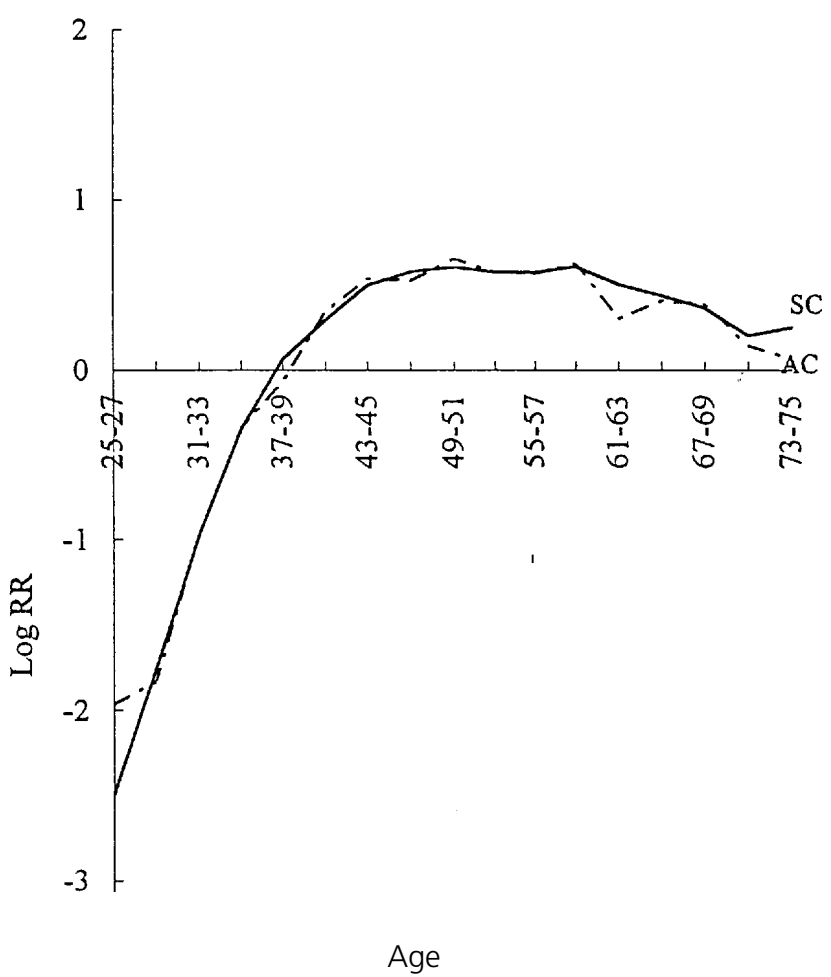

Fig. 1. Age effects on the incidence of squamous cell carcinoma (SC) and adenocarcinoma (AC) in Taiwan between 1979 and 1990.

cohort effect fluctuates and remains above the average, while the relative risks decrease sharply after the 1927 birth cohort for squamous cell carcinoma and the 1930 birth cohort for adenocarcinoma. However the risk remained at a plateau or slightly increased in most recent birth cohorts for both histologic types. The decrease was such that the 1927 cohort has a risk of squamous cell carcinoma 7.8 times larger than the 1948 cohort, whereas the 1930 cohort has a risk of 


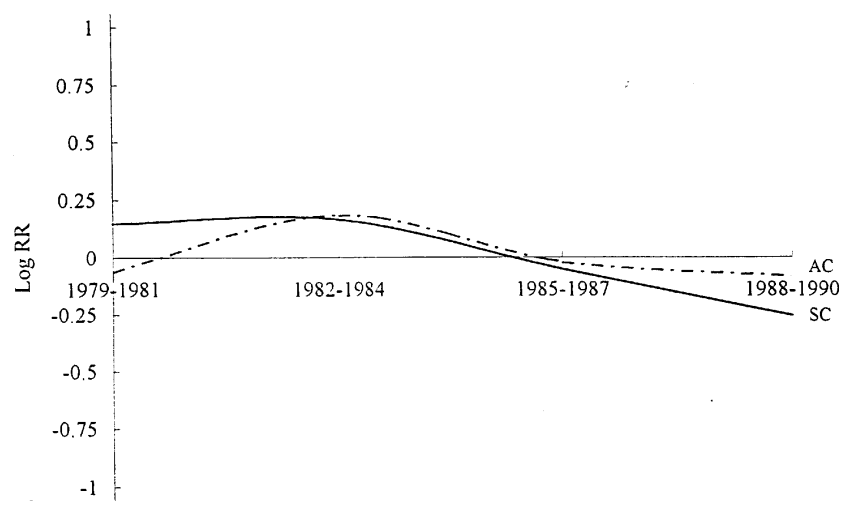

Calender Year

Fig. 2. Period effects on the incidence of squamous cell carcinoma (SC) and adenocarcinoma (AC) in Taiwan between 1979 and 1990.

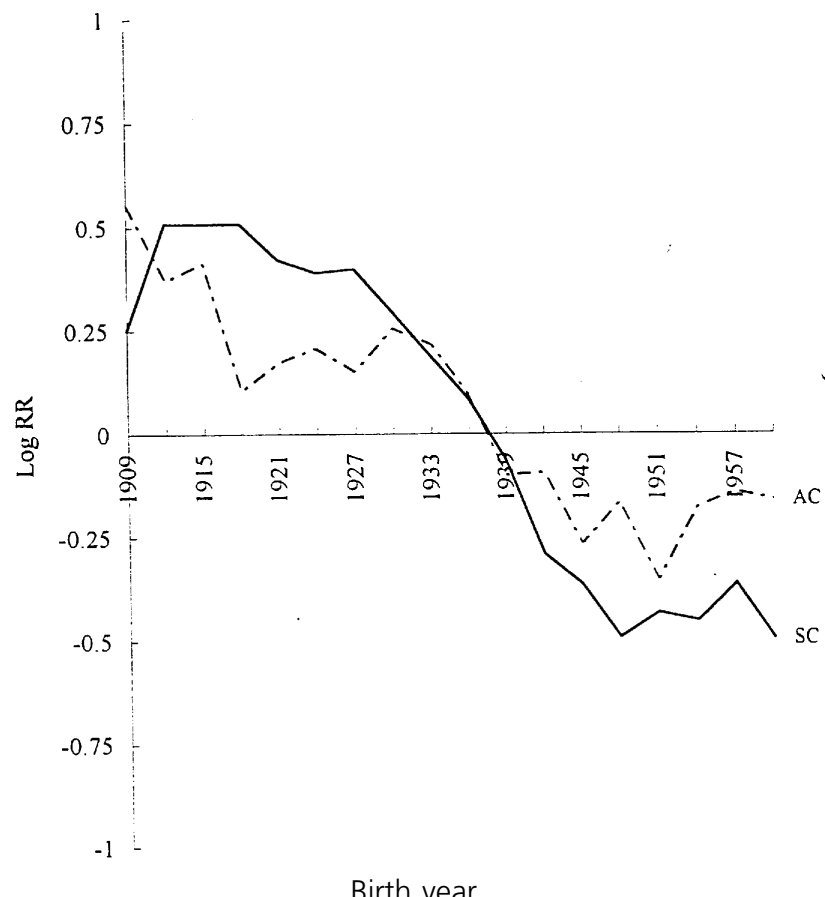

Fig. 3. Cohort effects on the incidence of squamous cell carcinoma (SC) and adenocarcinoma (AC) in Taiwan between 1979 and 1990.

adenocarcinoma 4.9 times larger than the 1951 cohort. Consequently, the cohort effects are much greater for squamous cell carcinoma than for adenocarcinoma.

\section{Discussion}

Time trends of incidence rate for a particular disease can provide an epidemiologist with valuable clues or hypotheses for disease etiology ${ }^{(21)}$. Three temporal factors which are often considered in such an investigation are age, period (year of diagnosis) and cohort (year of birth). In 1939, Frost first employed these three factors on mortality rates from tuberculosis in Massachusetts ${ }^{(22)}$. This technique was further adopted by Case to establish the value of cohort analysis ${ }^{(23)}$. Nevertheless the technique remained a graphical one and the contributions of various time factors determined visually to examine patterns in disease rates over time ${ }^{(24)}$. In contrast, statistical APC analysis has been developed in an attempt to overcome these drawbacks and to quantify the separate effects of the age, period and cohort variables, provided suitable constraints are imposed ${ }^{(25)}$. The constraints proposed by Osmond and Gardner were determined solely within the data and yielded an objective indication of the statistical significance of a particular pattern ${ }^{(16)}$. The limitation of this technique was subsequently pointed out by Holford, and a more objective method among the APC models proposed ${ }^{(21)}$.

In applying APC analysis to incidence trends in the present study, all three temporal effects revealed useful information. The age effects reflect largely the change of the biological aging process ${ }^{(26)}$. The peak of the age effect for both histotypes occurs at the same age group of 49-51 but, after this period, there was for the case of squamous cell carcinoma, a small decline in age effect with age whereas the age effect for adenocarcinoma showed a clear decline with increasing age. It is possible that the occurrence of both histotypes is influenced by female sex hormones so that incidence stops linear increase after menopause. This explanation is consistent with the patterns observed elsewhere, including UK, Finland and Norway ${ }^{(27)}$. However, such an age effect pattern is substantially different from that reported by Anton-Culver et al. ${ }^{(9)}$, who found continued risk of increase with age for both types of tumors. This departure could result from the authors lack of adjustment for the effects of period and cohort. Furthermore, it can be seen in Fig. 1 that the age effect shows a greater extent of decrease for adenocarcinoma than for squamous cell carcinoma after so-called 'age at menopause'. This discrepancy may, at least in part, reflect a more relevant role of female sex hormonal factors in adenocarcinoma than in squamous cell carcinoma carcinogenesis. These findings are consistent with the hypothesis of Hahnel's et al. ${ }^{(28)}$ which holds that cervical tissues are responsive to estrogens, and cervical adenocarcinoma appears to be more responsive than squamous tumors. However, some factors which may affect the decreased incidence after menopause, such as change in the sexual behavior and cohort effects etc, were considered less important in the present study, because we used the APC model to quantify the separate effects of the age, 
period and cohort variables on the incidence rates, adjusting for the temporal effects.

The distinction between the squamous cell carcinoma and adenocarcinoma was also reflected in the differing period effects. There was a substantially decreased risk of squamous cell carcinoma during the period from 1979-81 to 1988-90, while the period effect discovered in adenocarcinoma was also decreased but to a lesser extent between the period 198587 and 1988-90. Our data is supported by Eide ${ }^{(4)}$, who suggested that squamous cell carcinoma are more susceptible to time trends than adenocarcinoma. Interpretation of these findings is complicated by the fact that it is more difficult to detect preinvasive stage of adenocarcinoma than squamous cell carcinoma by screening practices ${ }^{(29)}$ since a generally accepted rationale for this belief holds that adenocarcinoma occurs high in the endocervix canal and is therefore hidden from early diagnosis by Papanicolaou smear. Furthermore, some factors which may affect the period effects, such as improvements in diagnosis over time especially for adenocarcinoma, poorer diagnosis for older women, prevalence of hysterectomy and registration practices, might affect the number of cases diagnosed as cervical cancer and bring forth a misleading time trend. Thus, it is possible that differences in period effect patterns between squamous cell carcinoma and adenocarcinoma do not reflect etiologic distinctions, but more trivial period factors. Continued surveillance is warranted, however, with special attention to the trends in adenocarcinoma.

In addition to age and period effects, the cohort phenomenon in this study was the most intriguing finding. This may imply that some important determinants of the disease occur in early life, which express their effects some time later. In discussing the determinants of a birth cohort phenomenon of a disease, one should always consider the possible influences of dietary habits, environmental status, war experiences, epidemics, etc in the early life of the population studied ${ }^{(24)}$. In this study, cohort trend analyses indicate a steady moderation of both histotype risk with the advancing birth cohorts. The largest decrease in slopes was the 1927-48 birth cohort for squamous cell carcinoma, while those in adenocarcinoma was for the 1930-51 cohort but to a smaller slope. What happened to the 1927-51 birth cohort in their early stages of cervical cancer development? It is likely that women born during these cohorts spent part of their child-bearing lives during the 1960-80 family planning and birth control campaign in Taiwan, when the fertility rate and birth rate decreased sharply since the total fertility rate had fallen rapidly from
1960-80 in Taiwan: 5.75 in 1960 and 2.50 in 1980. The rate was almost fixed around 2.50 in the early 1980s, and then showed a gradually declining trend, and in 1992 it was $1.80^{(30)}$. Therefore, it is noted that the 192751 cohort, in our analysis, came into child-bearing age right in the period of the 1960s to the 1980s. This coincidence in time may imply that the reproductive factors are one of the most likely possible sources of the cohort effect. Furthermore, the cohort effect discovered in our APC analysis is much greater for squamous cell carcinoma than for adenocarcinoma (Fig. 3). This may reflect more a relevant role of parity in squamous cell carcinoma than in adenocarcinoma carcinogenesis. This idea was supported by Brinton et al. ${ }^{(31)}$, who reported that women with multiparity had excess risks for both squamous cell carcinoma and adenocarcinoma, but the relationship was significant only for the former. Moreover, one of the hypotheses related to the development of cervical cancer resulting from the laceration of the cervix during childbirth was supported by our previous study ${ }^{(32,33)}$, as we observed a linear relationship between CIN risk and a number of vaginal deliveries, with women reporting four or more vaginal deliveries being at a twofold excess risk compared with women with one or no vaginal deliveries, even when adjusting for potential confounding factors.

In conclusion, we have demonstrated that ageperiod-cohort patterns of squamous cell carcinoma differs etiologically from that of adenocarcinoma, that adenocarcinoma appears to be more responsive to female sex hormones than squamous cell carcinoma after menopause as the determinant of the differing age effects, that cytologic screening has less effect on preventing adenocarcinoma than squamous cell carcinoma as the determinant of the differing period effects, and the reproductive pattern changes is more relevant to squamous cell carcinoma than in adenocarcinoma as the determinant of the differing cohort effects. Although there is the possibility of a gap between epidemiologic characteristics and theoretical cancer models, our findings may help to develop etiologic hypotheses and support the proposal of a variable etiology between both histologic types.

\section{Acknowledgments}

We thank Dr J.M. Weaver for many valuable comments and Mr W.T. Ho for technical assistance.

\section{References}

1 Saigo PE, Cain JM, Kim WH, Gaynor JJ, Johnson K, Lewis JL. Prognostic factors in adenocarcinoma of the uterine cervix. Cancer 1986; 57: 1584-93. 
2 Teshima S, Shimosato Y, Kishi K, Kasamatsu T, Ohmik, Uei Y. Early stage adenocarcinoma of the uterine cervix: Histopathologic analysis with consideration of histogenesis. Cancer 1985; 56: 167-72.

3 Silcocks PBS, Thornton-Jones H, Murphy M. Squamous and adenocarcinoma of the uterine cervix: A comparison using routine data. Br J Cancer 1987; 55: 321-5.

4 Eide J. Cancer of uterine cervix in Norway by histologic type, 1970-84. J Natl Cancer Inst 1987; 79: 199-203.

5 Hopkins MP, Morley GW. A comparison of adenocarcinoma and squamous cell carcinoma of the cervix. Obstet Gynecol 1991; 77: 912-17.

6 Devesa SS, Young JL, Brinton LA, Fraumeni JF. Recent trends in cervix uteri cancer. Cancer 1989; 64: 2184-90.

7 Peters RK, Chao A, Mack TM, Thomas D, Bernstein L, Henderson BE. Increased frequency of adenocarcinoma of the uterine cervix in young women in Los Angeles County. J Natl Cancer Inst 1986; 76: 423-8.

8 Chilvers C, Mant D, Pike MC. Cervical adenocarcinoma and oral contraceptives. Br Med J 1987; 295: 1446-7.

9 Anton-Culver H, Bloss JD, Bringman D. Comparison of adenocarcinoma and squamous cell carcinoma of the uterine cervix: a population-based epidemiologic study. Am J Obstet Gynecol 1992; 166: 1507-14.

10 Barrett JC. Age, time and cohort factors in mortality from cancer of the cervix. J Hyg 1973; 71: 253-9.

11 Stevens RG, Moolgavkav SH, Lee JAH. Temporal trends in breast cancer. Am J Epidemiol 1982; 115: 759-77.

12 Moolgavkar SH, Stevens RG. Smoking and cancers of bladder and pancreases: risk and temporal trends. I Natl Cancer Inst 1981; 67: 15-23.

13 Roush GC, Holford TR, Schymura MJ, White C (eds). Cancer Risk and Incidence Trends - The Connecticut Perspective. Washington DC: Hemisphere Publishing 1987.

14 Robertson C, Boyle P. Age, period and cohort models: the use of individual records. Stat Med 1986; 5: 529-38.

15 Clayton D, Schiffler E. Models for temporal variation in cancer rates II: age-period-cohort model. Stat Med 1987; 6: 469-81.

16 Osmond C, Gardner MJ. Age, period and cohort models applied to cancer mortality rates. Stat Med 1982; 1: 245-59.

17 Department of Health, the Executive Yuan. Cancer Registry Annual Report in Taiwan Area, 1990. Taipei: Department of Health, Executive Yuan, 1994.
18 World Health Organization, ICD-O. International Classification of Diseases for Oncology, Geneva: WHO, 1976.

19 Ministry of Interior, R.O.C.: Demographic Facts, 1974-1992. Taipei: Ministry of Interior, R.O.C., 1975-93.

20 SAS Institute Inc.: SAS/IML: User's Guide, Release 6.04 Edition. Cary, NC: SAS Institute Inc, 1988.

21 Holford TR. The estimation of age, period and cohort effects for vital rates. Biometrices 1983; 39: 311-24.

22 Frost $\mathrm{WH}$. The age selection of mortality from tuberculosis in successive decades. Am J Hyg 1938; 70: 91-6.

23 Case RAM. Cohort analysis of mortality rates as an historical or narrative technique. Br J Prev and Soc Med 1956; 10: 159-71.

24 MacMahon B, Terry WB. Application of cohort analysis to the study of time trends in neoplastic disease. J Chron Dis 1958; 7: 24-35.

25 Kupper LL, Janis JM, Darmous A, Greenberg BG. Statistical age-period-cohort analysis: a review and critique. J Chron Dis 1985; 38 (Suppl. 811).

26 Doll R. The age distribution of cancer: implications for models of carcinogenesis. J Roy Stat Soc 1971; 134: (Suppl. 133): 133-66 (Abstract).

27 Armstrong B. Endocrine factors in human carcinogenesis. In: Bartsh $\mathrm{H}$, Armstrong $\mathrm{B}$ eds. Host Factors in Human Carcinogenesis. Lyon: IARC Scientific Publications No. 39. 1982; 193.

28 Hahnel R, Martin JD, Masters AM. Estrogen receptors and blood hormone levels in cervical carcinoma and other gynecological tumors. Gynecol Oncol 1979; 8: 226-33.

29 Kim K, Rigal RD, Patrick JR. The changing trend of uterine cancer and cytology. A study of morbidity and mortality trends over a twenty-year period. Cancer 1978; 42: 2439-49.

30 Taiwan Provincial Department of Health. Vital Statistics, 1950-1992. Chung-Hsing Village, Taiwan: Provincial Department of Health, 1951-93.

31 Brinton LA, Tashima KT, Lehman HF, et al. Epidemiology of cervical cancer by cell type. Cancer Res 1987; 47: 1706-11.

32 Wang PD, Lin RS. Risk factors for cervical intraepithelial neoplasia in Taiwan. Gynecologic Oncology 1996; 62: 10-18.

33 Wang PD, Lin RS. Epidemiology of cervical cancer in Taiwan. Gynecologic Oncology 1996; 62: 344-52.

Accepted for publication November 12, 1996 